form of abrupt halt, who, for years, was in constant dread of sudden death. In spite of $\mathrm{my}$ frequent assurances that her stomach and not her heart was the viscus affected, she could not quite believe that her former medical advisers were all wrong, and I alone right, for, "can I not feel my heart stop?" she would often say. During one of her trips north, I prevailed on her to consult that chief among diagnosticians, DaCosta. Since that time, ten years ago, she has learned that flatulence by pressure on the tendinous portion of the diaphragm can cause tumultuous action of the heart as well as complete intermittency.

Dr. Dehio of Dorpat claims that most cases of bra. dycardia are due to nutritional changes in the auto. matic nerve centers in the heart, dependent probably on local sclerosis of the branches of the coronary arteries leading to such centers. As shown by Dr. J. W. Ogle (London Lancet, February, 1898) in four cases reported by him to the London Pathological Society, the bradycardia was associated with thickening of the endocardium and fibrinous deposits mostly at the bases of the ventricles. The bradycardia of true angina pectoris is nearly always associated with sclerosis, calcification or ossification of the coronary arteries themselves, or else a similar condition at or near the aortic opening. This condition renders the prognosis very grave, and calls for promptness in lowering arterial tension, in toning up the cardiac musculature, and in anticipating cerebral ischemia. The prompt administration of such remedies as nitrite amyl, trinitrin and strychnia, with the continued use of the iodids, fulfil such requirements. All vasoconstrictors, such as ergot, digitalis and quinin, should be studiously avoided. Morphia should be given, if at all, with the greatest caution.

In this connection it might be well to call attention to the cardiac ganglia imbedded in the auricles, These ganglia are intimately connected with the sym. pathetic nervous system. They are purely sensory, and have no motor fibera. Romberg and His have demonstrated in the heart muscular transition fibers that pass directly from the auricles to the ventricles, and the rhythmic action of the heart is now believed to be entirely dependent upon the contraction of these muscular fibers, and is not due, as was generally held, to the cerebrospinal or sympathetic systems. The action of the heart is therefore automatic, Uremia is responsible for a considerable number of cases of bradycardia. These cases are markedly benefited by rest, a milk diet, warm baths and abundance of pure water. The great majority of cases of bradycardia are due to diseases of the cord and brain. In these cases the prognosis is hopeless. The prognosis in other cases depends upon the cause-toxemias, neuroses, reflex, and convalescence from various diseases, etc.

The cause, taken in connection with the dynamics of the heart, should enable you to prognosticate accurately. Given a slow pulse, accompanied, upon slight exertion, by vertigo from cerebral anemia, cyanosis and dyspnea, your prognosis must of necessity be grave; but, on the other hand, as long as the slow, strong beats of the heart supply all wants of brain and body, the prognosis is favorable. However, it can be stated broadly, that arhythmia is usually of grave import. Occurring in children in connection with fever, it is strongly suggestive of tuberculous meningitis.

The unequal pulse always demands a guarded prog- nosis, as it usually denotes an aneurysm involving the root of the subclavian artery, although it may be due to obliteration of the radial by injury or by $\operatorname{som} \theta$ growth making pressure on the vessel. At other times it it purely neurotic.

It is beyond the scope of this paper to enter into details in reference to the many varieties of pulse and their significance. I have endeavored but to point the way and to remind all general practitioners of medicine that "the proper study of mankind is man," and the more thorough our study of man, the more competent and successful practitioners we will become. I would not dissuade any of the younger members of the profession from the alluring fields of bacteriology, for medicine is under lasting obligations to the careful students of the lower forms of life, but let us never forget that it is by the art and not by the science of medicine that we gain our reputation, and, incidentally, our livelihood. All can not be celebrated botanists, chemists, neurologists, bacteriologists and path. ologists. Let us take unto ourselves the homely proverb, ne sutor crepidam judicaret. By availing ourselves of the labors of these men in special fields we will profit greatly; but our success as practising physicians, now, as formerly, will depend upon our close observation of all that concerns homo bimanus.

Charles Darwin was astounded at the instantaneous and immense reputation his "Origin of Species" brought him. He tells us in his modest way that he had done nothing but observe closely; aye, there is the secret, he had observed, closely, accurately, scientifically, the minutest detail. As in natural history, so in medicine. How many of us have eyes, yet see not; ears, yet hear not? Shakespeare spoke truly, when he said: "Most poor things point to rich ends." There are men in our calling who have studied so carefully the stomach of the mosquito that they are able to tell you all the reasons why the malarial hematozoon prefers that particular viscus of that particular dipter to that of its gregarines or coccidia. This is all very interesting and scientific, but we do not treat gastric catarrh of the mosquito. By close observation of the laws of health and disease, we all may become qualified to practice the art of medicine successfully. It is a well-known fact that the the treatment of many diseases, notably pneumonia, tuberculosis, malaria and pertussis, has not been crowned with better results since the microscope has introduced to us the Lilliputian armies that cause their pathology; nor have the most successful practitioners been those best versed in the pure realms of science. The close observers have always, in every age, been the most successful physicians. With Oliver Wondell Holmes I say:

How blest is he who knows no meaner strife, Than art's long struggle with the foes of life.

\section{A. CASE OF ADIPOSIS DOLOROSA.}

Presented to the Section on Neurology and Medical Jurisprudence, at the Forty-ninth Annual Meeting of the American Medical Association, held at Denver. Colo., June 7-10, 1898.

BY AUGUSTUS A. ESHNER, M.D.,

PROFESSOR OF CLINICAL MEDICINE IN THE PHIL ADELPHIA POLYCLINIC; PHYSICIAN TO THE PHILADELPHIA hOSPITAL, ETC. PHILADELPHIA, PA.

In a paper read before the Philadelphia Neurological Society in 1891, Henry ${ }^{1}$ reported the case of a married woman, 63 years old, in whom, at the age of 
49 , somewhat circumscribed, painful, fatty swellings appeared successively in various parts of the body. The patient was an immoderate drinker and had had epileptiform convulsions frequently in infancy. Menstruation had begun at 11 and had ceased abruptly at 35. Sensibility was impaired in proportion to the deposition of fat. The case was described as one of myxedematoid dystrophy, and for the condition the designation paratrophy was proposed. In a paper read before the American Neurological Association in 1888 . Dercum ${ }^{2}$ reported the case of a widow, 51 years old, in whom the arms began to enlarge at the age of 48 or 49 and were the seat of pain. Back, shoulders, arms and sides of the chest became the seat of huge pendulous masses, firm yet elastic, in places finely lobulated and feeling like bundles of worms. Menstruation had begun at 15 and had ceased abruptly at 35. Microscopic examination of a bit of tissue removed from one of the swellings disclosed the presence of only fat and connective tissue. The case was described as one of subcutaneous connective-tissue dystrophy associated with symptoms resembling myxedema. In a subsequent communication Dercum ${ }^{3}$ reports in greater detail both of the foregoing cases, together with a third, describing them as "three cases of a hitherto unclassified affection resembling in its grosser aspects obesity, but associated with special nervous symptoms," and proposing for them the name of adiposis dolorosa. The third of these cases occurred in a widow, 60 years old, who had for many years had soft, fat-like masses or swellings in various parts of the body that were painful to touch. Cutaneous sensibility was diminished and the patient was demented. Death resulted from gradual failure of vital force and the thyroid gland was found to be enlarged, indurated and calcified. Death occurred also in the first of these cases and the thyroid was found likewise indurated and calcareous, although smail.

In the article on auliposis dolorosa in "A Text-book on Nervous Diseases by American Authors," Collins refers to six cases under the observation of Peterson and Loveland and to one under his own observation. He states that all of the cases but one occurred in women from 40 to 60 years of age; that in but one could a specific or alcoholic history be ruled out, and that in all a neuropathic predisposition was evident.

In a consideration of the treatment of myxedema, cretinism, exophthalmic goiter, obesity, etc., with thyroid extract, Ewaid ${ }^{5}$ refers to the case of a man, 47 years old, weighing 217 lbs., who presented a puffy appearance, with thick masses of fat about the nip. ples and the umbilicus and upon the neck, and pains resembling those of neuritis. Perspiration was scanty and the condition was suggestive of myxedema, though wanting in the characteristic features of this disorder.

Spiller ${ }^{6}$ has reported three cases of adiposis dolorosa, all in women, two unmarried and aged 25 and 65 respectively, and the other married and 45 years old. In the first there were both diffuse enlargement and distinct painful swellings, with paroxysmal exacerbations. Menstruation was normal. In the second case the patient began to grow stout at 40 , when menstruation ceased. For thirteen years there had been pain in the lower extremities, and for two years in the fatty

2 University Medical Magazine, December, 1888. p. 140.

American Journal of the Medical Sciences, 1892, p. 521.

Edited by Dercum. Philadelphia, 1895, p. 898.

Berliner klinische Wochenschrift, Jan. 21, 1898, p. 268

Medical News, Feb. 26, 1898, p. 268. tissue of the limbs and trunk, with paroxysmal exacerbations. The obesity had increased markedly since the advent of the latter pain. There were tenderness and pain on palpation, manipulation and pressure, and no anesthesia. The fatty accumulation was marked on the thighs, the calves, the abdomen, the nates and the back, and also in the arms, though less in the forearms and absent from the feet and the hands. There was no special deposit of fat and no pain in the face or neck. The isthmus of the thyroid was exceedingly small. The third patient had been corpulent for twelve or thirteen years, following the birth of her last child. Pain had been present for years, recurring in paroxysms. Swellings appeared beneath the skin of the lower extremities, at first small, there larger and finally diminishing in size. Sensibility was preserved and the face. was not involved. Im. provement followed massage and the administration of thyroid extract. A cross-section of musole from one of these cases showed no appreciable deviation from the normal, except perhaps a slight enlargement of the nuclei.

To this small number of cases I am able to add a further one, and also the notes of a case kindly placed at my disposal by Dr. Dercum:

M. G., a married white woman, 48 years old, was admitted to the medical department of the Philadelphia Hospital June 6,1896 , and shortly afterward the following facts were elicited: Her mother had been dropsical and had died at the age of 86 . Her father and one brother had been killed in war. Two other and older brothers were alive and well. A sister also was living at the age of 60 years. Another sister had died in a dropsical state. The sister and one brother were said to be stout, but within ordinary limits. A maternal great-grandmother also had been unduly stout. The patient related that she had suffered from attacks of erysipelas of the lower extremities on four different occasions, the first at about the age of 30 , the second six years later, the third at some forgotten time after, and the fourth a year before coming under observation. She had borne five children, all delivered instrumentally. Of these one had died during birth and another of diphtheria; of the remainder one boy and one girl were spare and a daughter of 28 was stout. Her husband had died of pneumonia eighteen years previously. Menstruation had begun at the age of 13 years and had been regular and painless until the age of 41 , recurring thereafter with increasing frequency and being attended with excessive hemorrhage. At the age of 35 the patient is said to have broken down from overwork and worry. At this time twitchings were observed, and a sense of such movements had persisted thereafter. After an interval of uncertain length the woman noticed enlargement of the abdomen. Then the lower extremities became involved, the increase in size extending gradually and progressively over the entire body, with the exception of the face and the hands and the feet. The patient had suffered a good deal from pains in the back, especially in the sacral and interscapular regions. The pain was intermittent and had set in with the accumulation of fat. For more than two years there had been a more or less constant sensation of beating in the head, and for a year or more ringing in the ears. There was no vertigo, and auditory perception was good. The appetite was preserved; it had never been excessive, and the patient had no preference for, nor had she indulged in, special kinds of food. There was at times nausea and occasionally vomiting, the ejected matters sometimes containing blood. For more than a year the patient had been helpless, being compelled to lie abed and unable to move about. She occupied two single beds in the hospital and was scarcely able to help herself in any way.

On examination at the time of admission to the hospital heart and lunge were found to present no abnormity. The face was scarcely larger or fuller than that of a person of ordinary size. The circumference of the neck was 17 inches, of the bust 60 inches, of the waist 53 inches, of the abdomen 70 inches, of the arm above the elbow 21.5 inches. The tempera. ture, taken for months at a time, adhered to the normal level ; the respirations fluctuated between 18 and 32 , averaging perhaps 24 ; the pulse ranged between 68 and 108 , averaging about 84. The urive. examined repeatedly, displayed a specific gravty between 1019 and 1028 and, other than the presence of a mall number of leucocytes, exhibited no deviation from the 
normal. On examination on June 18, 1897, the hands were ob- der. The fat of the neck was not markedly increased. The served to be well nourished, but of ordinary size. The circum- breasts were large and extensive, though flat, falling in buge ference of the forearms above the wrist was 7.2) inches, below apron-like folds. The right appeared to be larger than the left the styloid processes 6.6 inches. At the elbow there were and its pigmentation was the more pronounced. The mammillary marked creases on the dorsal and palmar aspects. The cir- areolie were marked, and also the tubercles. The circumfercumference 1 inch below the flexure was on the right $13 . \%$ ence of the trunk at the waist was 41 inches, at the level of the
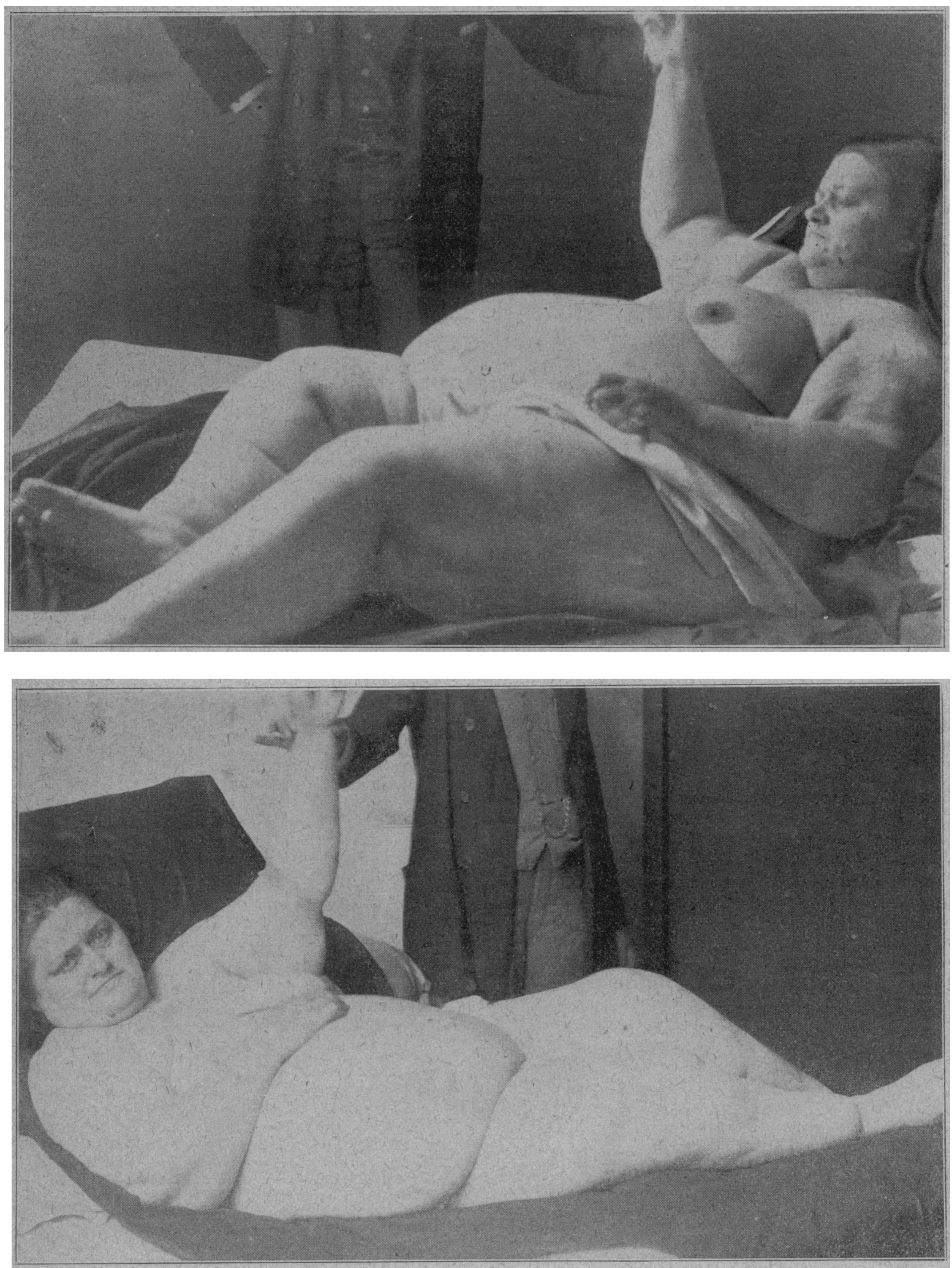

inches, on the left 12.5 inches. There was only a small growth sacrum 59 inches. The distance from the olecranon process of of hair in the axill:u. The mid-humeral circumference on the the ulna to the styloid process was 10 inches, from the acroright was 16.5 inches, on the left 16 inches. The upper extrem- mion to the olecranon 13.75 inches. The circumference at the ity was strikingly conoidal in form, with the base at the shoul- middle of the thigh was 31 inches. The distance from the outer 
margin of the patella to the external malleolus was 16 inches and to the outer extremity of the inguinal fold 17 inches. The circumference at the middle of the leg was 17.5 inches. The right foot was 9.75 inches long, the left 10.25 inches. The surface of the skin. was mottled, purplish and cyanotic on the right side of the body, on which the patient lay. The heart-sounds were quite clear and distinct. The tongue was broad, fissured and tremulous; the patient stated that it had always presented a similar appearance. The ears were not enlarged. The thyroid gland could not be felt. The hair was soft and unctuous. The skin also was soft, and perspiration was free. The masses of fat yielded a cord-like sensation to touch, and manipulation elicited tenderness. The patient had received varied treatment, without appreciable effect. For a time desiccated extract of thyroid gland was prescribed, 5 grains twice daily, but in a short time the patient declined to take the medicament, complaining, among other things, that she had been poisoned by some of the drugs she had received. Subequently she developed pronounced delusions and it became necessary to remove her to the department for the insane. ladder and wounded the right leg above the ankle with a rusty nail. Symptoms of lymphangitis developed, from which recovery ensued. Three months later the left leg was wounded as the result of a fall and it became inflamed and increased in size. The enlargement extended upward, and in a short time the right leg also began to increase in size. The hyperplastic parts became the seat of cracks and fissures and indolent recurring ulcers. The illustrations accompanying the report show great enlargement of the legs, thighs and abdomen, with immense buttocks, full shoulders, while the face, feet and hands were relatively small.

As an instance of a further anomalous form of obesity may be mention a case reported by Adler, ${ }^{8}$ occurring in a mulatto, 53 years old, with a syphilitic history,

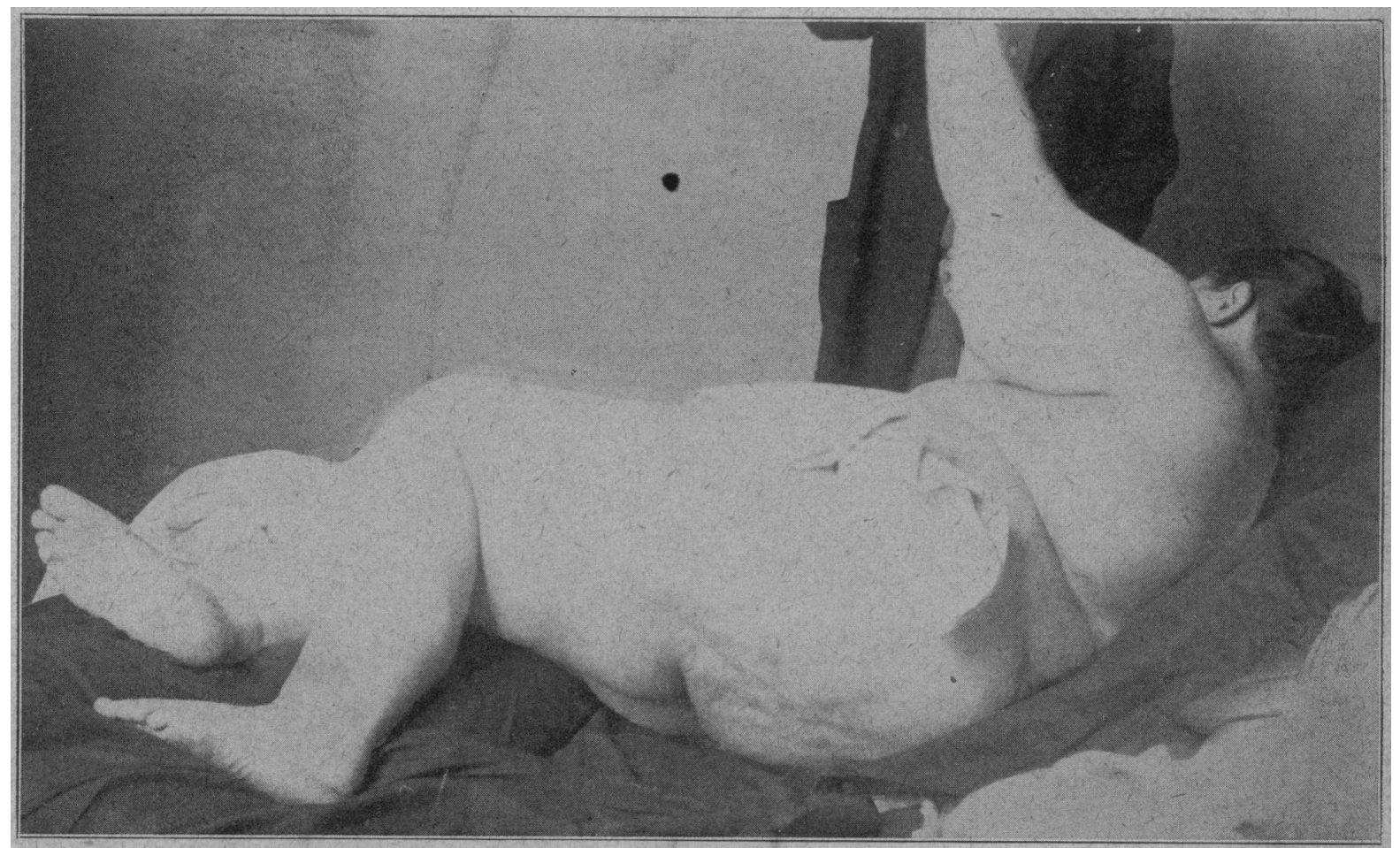

While it may be objected that this case is lacking | but with none of obesity in the family, who presented in some of the symptoms of adiposis dolorosa, as laid an enormous growth in the middle line at the back of down by Dercum, it will be conceded that it is unlike the neck, two smaller biluteral masses symmetrically an ordinary case of obesity. The principal accumu- situated in the occipital region, another in the middle tions or deposits are nearest the trunk and they hang line below the chin forming an immense dewlap and in conspicuous folds, while the degree of incapacita- extending up into the parotid regions, one in each tion for the simplest activity is extreme. Pain has groin absolutely symmetrical and projecting forward not been a prominent symptom and we have no knowl- as well-rounded swellings. A more diffuse fatty deedge of paroxysmal variations in the degree of enlarge- posit was present on the arms and forearms. There ment, but the patient's memory was not reliable and was one unilateral tumor over the left rectus muscle her statements were at times wanting in positiveness. above the level of the umbilicus. The several growths The case is in some respects not unlike one reported were movable and free from pain, while the overlying by Laidlaw ${ }^{\top}$ as an example of elephantiasis, occurring skin was tense and unadherent.

in a woman, 45 years old, who in the performance of some laborious task at the age of 27 , suffered an injury of the abdomen near the umbilicus. The cutaneous and the subcutaneous tissues of the affected parts became reddened, swollen and infiltrated. The acute symptoms soon subsided, leaving well-marked hypertrophy, which gradually diminished. Two years later the left leg became covered with scales and enlarged, and the abdominal condition grew gradually worse. Seven years after this the woman fell from a

I am indebted to Dr. Dercum for the following notes:

A married woman, 36 years old, in excellent health and free from physical abnormity, while standing in the aisle of a trolley car, was thrown to the floor as the result of a collision with a heavy wagon and at once became unconscious. She was sent home in a carriage and was subsequently unable to move without assistance. Later she was able to sit up in a rolling-chair and got about with the aid of crutches. A miscarriage took place fourteen days after the accident. The patient suffered greatly from pain. When examined, a year after the accident,
she could not stand without assistance, although she could 
walk with the aid of crutches. Superficial pressure over the spine in the lower doreal, lower lumbar and sacral regions and also over the coccyx induced pain, and deep pressure over the muscles of the back of the trunk in the lower dorsal and lumbar regions on the left side, and in less degree on the right side, elicited tenderness. Similar tenderness was noted also on the left side of the chest. Transmitted shock caused pain referred to the lumbar region, flexion of the trunk pain referred to the same region, and especially lateral flexion to the left. A ttempte to extend the legs, with the patient seated on a chair, induced pain referred to the back of the legs and thighs. The knee-jerks were exaggerated. There was some analgesia of the left arm, left $l e g$ and left side of the trunk, but no inflammatory or ovarian tenderness, and no limitation of the visual fields. Upon the left forearm there were found seven or eight small swellings, varying in size from a small marble to a walnut, soft to the touch and apparently made up of fatty tissue. These were exceedingly painful and tender on pressure. The patient was positive that they had made their appearance some six weeks after her accident, when she became aware of the presence of several slightly swollen and tender places upon the left forearm. These had increased in size and continued to be painful. The patient suffered from occipital headache, from a sinse of constriction of the head, from vertigo, at times from ringing in the ears and from backache, relieved by the recumbent posture. She slept badly, having difficulty in getting to sleep and being disturbed by frightful dreams. She felt worse in the morning on arising than at night on retiring. She suffered from indigestion and chronic constipation. Palpitation of the heart was frequent, especially after unpleasant dreams. Micturition was increased in frequency, particularly at night. Perspiration was induced by slight exertion. On examination, some nine months later, it was found there had been some subeidence of the symptoms indicative of spinal strain. The painful masses upon the left forearm were still present and had undergone no appreciable change in eize. There were now noticed, in addition, several large, diffuse swellings upon the lower portion of the back and in the sacral region. These were soft and painful and tender on pressure, and like the others, although larger.

As defined by Dercum, ${ }^{9}$ adiposis dolorosa is a disorder characterized by irregular, sometimes symmet. rical, deposits of fatty masses in various portions of the body, preceded or attended with pain. It appears about middle life or later and the larger number of cases have occurred in females. In some there been an alcoholic history, in others a syphilitic history; in still others rheumatism may have been an etiologic factor; further, traumatism may be a provocative agency. Nothing more definite, however, can be said of the etiology. The principal manifestation is the presence of masses of fatty tissue of variable size, sometimes exceedingly large, variously distributed upon the trunk and the extremities, and the seat of pain sometimes spontaneous, sometimes induced by pressure or manipulation. The affection may set in with pain of neuritic character and the swellings may undergo increase of size in paroxysms attended with exacerbations of pain. The new fatty tissue has a boggy, soft, at times pultaceous and wormlike, feel. Face, hands and feet are not involved. In some cases sensibility is impaired. The skin exhibits no gross changes. Hemorrhages from mucous surfaces have been observed in some cases. The disease is essen. tially chronic, with a tendency to be progressive in course. The morbid anatomy consists, so far as is known, in an increase of fatty and connective tissue, with degenerative changes in nerves. The thyroid gland has been found indurated and calcareous. The disorder differs from other forms of obesity in the presence of pains and sometimes of impaired sensibility; and from myxedema in the freedom of face, hands and feet and in the absence of the pronounced mental and trophic manifestations of that disease. In treatment good results may be expected from administration of thyroid extract and from massage.

\footnotetext{
${ }_{9}^{9}$ Twentieth Century Practice, vol. xi, p. 554.
}

\section{SOME CONSIDERATIONS OF THE SYMP. TOMATOLOGY IN THE DIAGNOSIS OF TABES.}

Presented to the Section on Neurology and Medical Jurisprudence, at the Forty-ninth Annual Meeting of the American Medical Association, held at Denver. Colo., June 7-10, 1898.

BY J. M. AIKIN, M.D.

Neurologist to the Methodist Hospital, and Clinical Lecturer on Diseases of the Brain and Nervous System, in the Creighton Medical College. OMAHA, NEB.

About two years since, a physician doing a large practice in general medicine, impressed me wonderfully with the statement that seven cases of locomotor ataxia had come under his observation and treatment during the preceding three months. Of these, five had completely recovered, one died after three weeks' illness, from inability to swallow food, and one was convalescent. My disbelief in the curability of tabes induced me to seriously consider the alleged prevalence of the malady as truly alarming, whereupon I began investigating vital statistics from several of the large cities of the United States, and found that in the whole number of deaths reported as due directly to some form of nervous disease, only about 1.2 per cent. was accredited to locomotor ataxia. Reviewing some cases neurologic in character, that I was permitted to observe and treat during the five years preceding 1897, the shortest period for observation in any one case being one year, I was unable, in seventeen out of twenty-thres alleged cases of locomotor ataxia, to verify the diagnosis.

The observation of symptoms in disease is quite as necessary as the accumulation of evidence in a legal case, and a knowledge of their meaning is certainly no less imperative in forming right conclusions, than is the action of a judicial mind in estimating the value of testimony in a case at law. Moreover, clinical evidence in disease is quite as truthful as testimony at the bar, while microscopic findings certainly can not be more liable to error than twelve jurors, each exposed to ulterior influences.

During the past few years, the surprising advance of medical minds in the pathogenesis of nerve tissue per se is without a parallel in any branch of medicine, or period in its history. I therefore proceedwith a confidence maintained not alone by self-gleaned facts, but supported by truths, the authenticity for which many medical men in this Association deserve honorable mention - to assume that neurologic medicine has established a basis from which a proper estimate of the evidence, in at least the more common diseases of the nervous system, may render serious mistakes in diagnosis unpardonable.

The activity shown by American physicians recently, in placing before the public so many excellent text-books and essays, treating especially of the features of nervous diseases, gives assurance irrefutable that those already in active practice have easy access to the latest information on this class of diseases.

The American propensity to observe, and inquire into the meaning of the thing seen, has placed him as a leader of the world in his observation and knowledge of the phenomena of the nervous system. Clinical evidences in individual instances of nervous diseases presenting varying symptoms, are accumulating and must accumulate until, by comparison and elimination, we arrive at the characteristics of a type, as has years since been done by internal medicine in 